\title{
Myocardin regulates expression of contractile genes in smooth muscle cells and is required for closure of the ductus arteriosus in mice
}

\author{
Jianhe Huang,,1,2 Lan Cheng,, ${ }^{1,2}$ Jian Li,1,2 Mary Chen,1,2 Deying Zhou,1,2 Min Min Lu, 1,2 \\ Aaron Proweller, ${ }^{1,2}$ Jonathan A. Epstein, ${ }^{1,2,3}$ and Michael S. Parmacek ${ }^{1,2}$ \\ 'Department of Medicine, ${ }^{2}$ Cardiovascular Institute, and ${ }^{3}$ Department of Cell and Developmental Biology, \\ University of Pennsylvania School of Medicine, Philadelphia, Pennsylvania, USA.
}

\begin{abstract}
Myocardin (Myocd) is a potent transcriptional coactivator that has been implicated in cardiovascular development and adaptation of the cardiovascular system to hemodynamic stress. To determine the function of myocardin in the developing cardiovascular system, $\mathrm{Myocd}^{F / F} / \mathrm{Wnt1}-\mathrm{Cre}^{+}$and $\mathrm{Myocd}^{F / F} / \mathrm{Pax}^{-\mathrm{Cre}^{+}}$mice were generated in which the myocardin gene was selectively ablated in neural crest-derived SMCs populating the

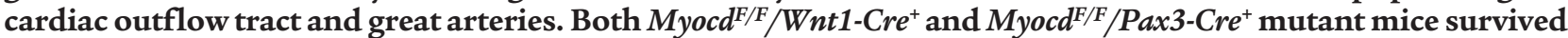
to birth, but died prior to postnatal day 3 from patent ductus arteriosus (PDA). Neural crest-derived SMCs populating the ductus arteriosus (DA) and great arteries exhibited a cell autonomous block in expression of myocardin-regulated genes encoding SMC-restricted contractile proteins. Moreover, Myocd-deficient vascular SMCs populating the DA exhibited ultrastructural features generally associated with the SMC synthetic, rather than contractile, phenotype. Consistent with these findings, ablation of the Myocd gene in primary aortic SMCs harvested from Myocd conditional mutant mice caused a dramatic decrease in SMC contractile protein expression. Taken together, these data demonstrate that myocardin regulates expression of genes required for the contractile phenotype in neural crest-derived SMCs and provide new insights into the molecular and genetic programs that may underlie PDA.
\end{abstract}

\section{Introduction}

Congenital heart disease is the most common cause of infantile deaths from birth defects in the United States. One of the most common congenital heart defects, affecting approximately 1 in 2,000 live-born infants is patent ductus arteriosus (PDA) (1). Most frequently observed in preterm infants, PDA is a condition generally responsive to treatment with cyclooxygenase inhibitors (2). In full-term infants, environmental exposures and maternal infection predispose to PDA (3-5). However, there is emerging evidence that PDA in full-term infants may frequently have a genetic basis $(6,7)$. Familial PDA has been observed in Char syndrome, which is caused by a mutation in TFAP2B, encoding a helix-span-helix transcription factor expressed in the cardiac neural crest (8-10). More recently, French and American kindreds have been identified with heritable PDA and thoracic aortic aneurysms caused by mutations in the smooth muscle myosin heavy chain gene (MYH11) (11).

The ductus arteriosus (DA) is a muscular artery derived from the sixth left aortic arch artery. The DA directs blood flow from the pulmonary artery to the aorta, shunting blood away from the embryonic lungs. The pulmonary orifice of the DA is located immediately to the left of the bifurcation of the pulmonary trunk. The aortic end of the DA lies just beyond the origin of the left subclavian artery. PGE2, a potent vasodilatory prostaglandin, plays a critical role in maintaining the open status of the DA during fetal life (12). Normally, the DA is occluded shortly after birth, estab-

Nonstandard abbreviations used: Ad, adenovirus; DA, ductus arteriosus; MRTF-A, myocardin-related transcription factor A; PDA, patent DA; SMA, SM- $\alpha$-actin; SM-MyHC, SM-myosin heavy chain.

Conflict of interest: The authors have declared that no conflict of interest exists. Citation for this article: J. Clin. Invest. 118:515-525 (2008). doi:10.1172/JCI33304. lishing discrete pulmonary and systemic circulations. In humans DA closure actually begins in the second trimester of pregnancy, with formation of an intimal cushion composed of synthetic SMCs and abundant subendothelial extracellular matrix (ECM) $(6,13)$. At birth, with expansion of the lungs, the abrupt increase in oxygen tension inhibits ductal SMC voltage-dependent potassium channels, leading to an influx of calcium and ductal constriction (14-17). This is accompanied by a rapid decline in the level of circulating PGE2. Following functional closure, the ductus is anatomically obliterated when ductal SMCs secrete ECM and undergo apoptosis, resulting in fibrosis and formation of the ligamentum arteriosus.

Myocardin is a remarkably potent transcriptional coactivator expressed exclusively in cardiomyocytes and SMCs (reviewed in refs. 18, 19). The mouse myocardin gene (Myocd) encodes 2 protein isoforms, which are differentially expressed in cardiomyocytes and SMCs, respectively (20). The Myocd gene is expressed as early as E7.75 in the cardiac crescent, and subsequently Myocd is expressed in the embryonic heart and visceral and vascular SMCs $(21,22)$. Myocardin physically associates with the MADS box transcription factor serum response factor (SRF), activating a subset of genes implicated in cardiomyocyte and SMC differentiation (21-24). In ES cells, forced expression of myocardin transactivates most but not all genes encoding SMC contractile proteins (21). Myocardin-null (Myocd (-) $^{-}$) mouse embryos survive only to embryonic day E10.5, precluding assessment of the function of myocardin in late fetal and postnatal development (25). Myocd $^{-/-}$embryos exhibit a block in vascular SMC differentiation as well as defects in the yolk sac vasculature $(19,25)$. The primary cause of lethality in Myocd $^{-/-}$embryos is the subject of ongoing investigations. 
A

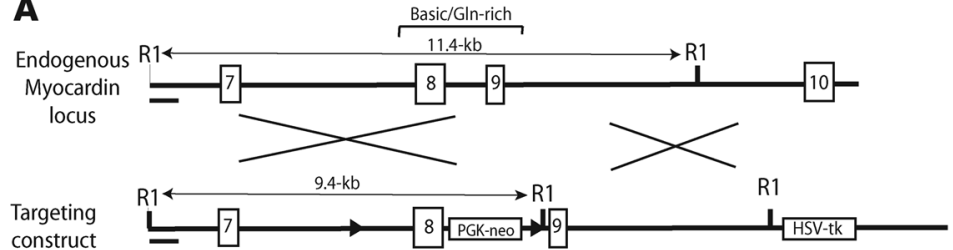

construct
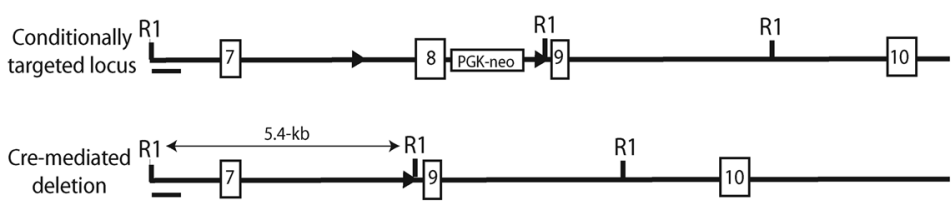

C

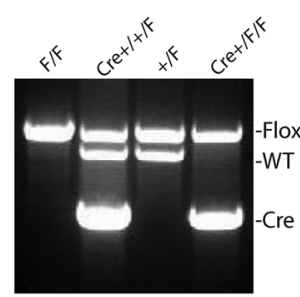

B

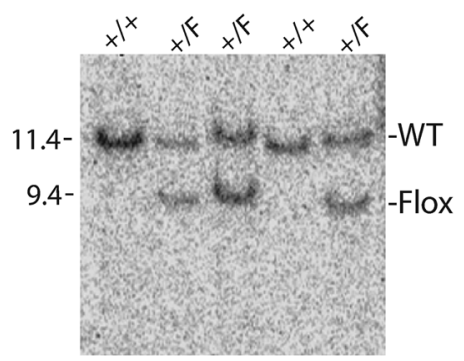

$\mathbf{E}$

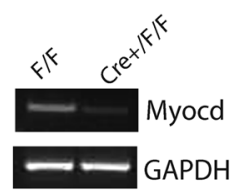

D

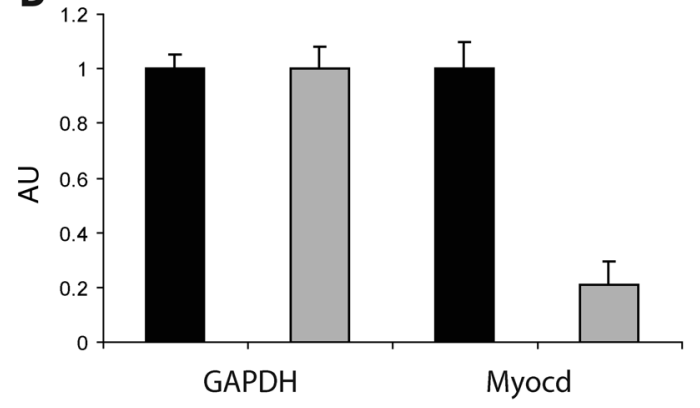

Figure 1

Conditional targeting of the Myocd gene. (A) Schematic representation of the Myocd gene showing exons 7-10 (rectangles). The basic and glutamine-rich domains are encoded by exons 8 and 9. EcoR1 sites (R1) are shown (top panel). The conditional gene-targeting construct contains the PGK-neomycin-resistance (neo) and HSV-thymidine kinase (tk) cassettes. LoxP sites (triangles) flank exon 8 (second panel). The conditionally targeted allele is depicted in the third panel. Targeted allele following Cre-mediated deletion. The position of the DNA probe used for Southern blot analyses is indicated below (black rectangle) (bottom panel). (B) Southern blot analysis of the targeted ES cells demonstrating the WT (11.4-kb) and flanked-by-loxP (Flox) (9.4-kb) alleles. Genotyping of WT (+/+), heterozygous (+/F), and homozygous (F/F) mice. (C) PCR genotyping of control floxed (F/F), control heterozygotes Wnt-Cre transgenic (Cre+/+/F), control heterozygote (+/F), and conditional mutant Wnt1-Cre transgenic (Cre+/F/F) mice. PCR was performed with primers PCR-A and PCR-B (see Methods) to identify products corresponding to WT (1,010-bp) and targeted (1,050-bp) alleles. Primer Cre800F and Cre800R were used to identify 800-bp Cre PCR product. (D and E) Myocardin gene expression in neural crest-derived arteries of $M y o c d^{F / F} / W n t 1-C r e^{+}$mutant mice. Real time RT-PCR was performed as described in Methods with mRNA harvested from the proximal aorta and carotid arteries of $4 \mathrm{P} 2 \mathrm{MyocdF/F/Wnt1-Cre+}$ mice (gray bars) and 4 control littermates (black bars). (D) Data are expressed as mean gene expression (arbitrary units) \pm SEM. (E) Representative DNA gel demonstrating GAPDH (291-bp) and myocardin (108-bp) amplified products in control (Cre-/F/F) and mutant ( $\left.\mathrm{Cre}^{+} / \mathrm{F} / \mathrm{F}\right)$ mice.

Myocardin is a member of a multigene family, which in metazoan species includes myocardin-related transcription factor-A (MRTF-A)/MKL1 and MRTF-B/MKL2 (reviewed in ref. 18). As with myocardin expression, forced expression of MRTF-A and MRTF-B transactivates SRF-dependent transcriptional regulatory elements controlling expression of SMC contractile genes (26). While all 3 MRTFs transduce cytoskeletal signals to the nucleus, it is noteworthy that myocardin is constitutively nuclear, while MRTF-A and MRTF-B translocate between the cytoplasm and the nucleus $(27,28)$. Mice harboring null or hypomorphic mutations in MRTF-B exhibit a spectrum of cardiovascular patterning defects, recapitulating common forms of congenital heart disease observed in humans (29-31). These patterning defects are attributable to a block in differentiation of cardiac neural crest cells into vascular SMCs. By contrast, MRTF- $A$ mutant mice are viable, but exhibit defects in myoepithelial cell differentiation, resulting in a block in the lactation cycle (32).

In the studies described in this report, the mouse Myocd gene was selectively ablated in the neural crest to examine the function of myocardin in vascular patterning and cardiovascular development. This conditional gene-targeting strategy isolates the function of myocardin in neural crest-derived SMCs from its function(s) in the heart and yolk sac vasculature. In contrast to myocardin-null mutants (Myocd-/-), which die prior to E10.5 (25), mice harboring a neural crest-restricted deletion of the myocardin gene $\left(\right.$ Myocd $^{F / F} /$ Wnt1-Cre ${ }^{+}$and $M y o c d^{F / F} /$ Pax3-Cre $\left.{ }^{+}\right)$are born in the anticipated Mendelian ratio, but die prior to P3 from PDA. At the molecular level, Myocd ${ }^{F / F} / \mathrm{Wnt1}_{-\mathrm{Cr} \mathrm{C}^{+}}$and $\mathrm{Myocd}^{\mathrm{F} / \mathrm{F}} / \mathrm{Pax} 3-\mathrm{Cre}^{+}$ pups exhibit a cell autonomous defect in expression of myocardinregulated genes encoding SMC contractile proteins. These studies demonstrate that myocardin plays a critical role in differentiation of neural crest-derived SMCs populating the great arteries as well as maintenance of the contractile SMC phenotype. Moreover, these studies provide a conceptual framework for understanding the molecular pathogenesis of PDA.

\section{Results}

Generation of mice containing a conditional null mutation of myocardin. To conditionally inactivate the Myocd gene, we created a modified allele in which exon 8 , encoding the basic and the $\mathrm{N}$ terminus of 


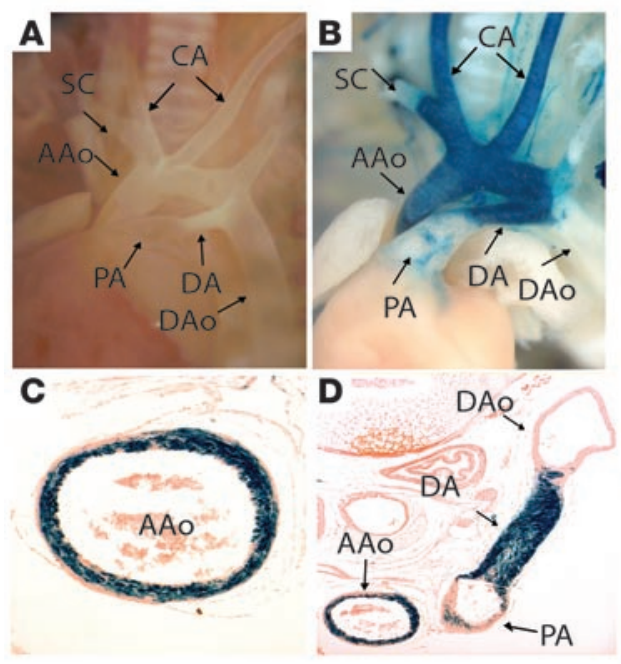

Figure 2

Wnt1-Cre mediated recombination in neural crest-derived SMCs. (A and B) Wnt1-Cre transgenic mice were interbred with R26R mice to define pattern of Cre-mediated gene excision. (A) P2 R26R+ control mouse demonstrating normal patterning of the cardiac outflow tract and great arteries, pulmonary artery (PA), DA, ascending aorta (AAo), descending aorta (DAo), carotid arteries (CA), and subclavian artery (SC). Original magnification, $\times 10$. (B) P2 Wnt1-Cre+/R26R+ mouse demonstrating $\beta$-galactosidase expression (blue stain) in arteries populated by neural crest-derived SMCs. Original magnification, $\times 10$. (C) Transverse section of P2 Wnt1-Cre+/R26R $R^{+}$mouse demonstrating the AAo. Most but not all SMCs populating the AAo stain blue. Original magnification, $\times 200$. (D) Transverse section of P2 Wnt1-Cre+/R26R+ mouse demonstrating robust Wnt1-Cre-mediated recombination in the DA. Original magnification, $\times 100$. (E and F) Frontal sections of an E11.5 Wnt1-Cre+/R26R+ embryo demonstrating $\beta$-galactosidase activity restricted to the endocardial cushions in the right ventricle $(R V)(F)$ and the $A A o$ and $D A(E)$. Original magnification, $\times 100$. (G-J) Immunohistochemical analyses performed with anti-myocardin antibody demonstrating markedly diminished myocardin expression (brown nuclear stain) in the SMCs populating the internal carotid artery (ICA) (G and $\mathbf{H}$ ) and aorta (Ao) (I and $\mathbf{J}$ ) of P2 MyocdFIF/Wnt1-Cre+ mutant mice ( $\mathbf{H}$ and $\mathbf{J})$ compared with a control Myocd ${ }^{F / F}$ littermate $(\mathbf{G}$ and I). Original magnification, $\times 300$ (carotid); $\times 400$ (aorta). the glutamine-rich domain of Myocd, is flanked by loxP (floxed) sites (Figure 1A). Exon 8 was targeted because the basic and glutamine-rich domains of myocardin are required for the association of myocardin with SRF and the capacity of myocardin to function as a transcriptional coactivator (22). In addition, cryptic splicing around exon 8 generates an out-of-frame truncated myocardin mutant protein. Correctly targeted ES cell clones were identified by Southern blot analyses (Figure 1B). ES cells containing a conditional mutation in the myocardin gene $\left(\right.$ Myocd $\left.^{F /+}\right)$ were injected into blastocysts to generate chimeric mice that transmitted the conditionally targeted Myocd allele through the germ line.

Homozygous myocardin floxed mice $\left(\right.$ Myocd $\left.^{F / F}\right)$ are viable, fertile, and generally indistinguishable from control littermates, demonstrating that the conditional gene-targeting strategy employed did not disrupt expression of the Myocd gene. To confirm that the gene-targeting strategy generated a true null allele, homozygous myocardin floxed mice $\left(M_{y o c d}{ }^{F / F}\right)$ were intercrossed with CMV-Cre transgenic mice that express high levels of Cre recombinase throughout the embryo (33). As anticipated, Myocd $^{F / F} / \mathrm{CMV}$-Cre ${ }^{+}$embryos recapitulate the phenotype of myocarotid arteries of $\mathrm{Myocd}^{F / F} / \mathrm{Wnt1}-\mathrm{Cr} \mathrm{e}^{+}$mutant mice was confirmed by immunohistochemical analyses (Figure 2, G-J).

In order to determine the precise spatial and temporal pattern of Cre-mediated gene excision, Wnt1-Cre transgenic mice were interbred with R26R indicator mice that express $\beta$-galactosidase in cells following Cre-mediated recombination (35). As anticipated, in P2 Wnt1-Cre ${ }^{+} / R 26 R^{+}$mice, blue staining indicative of $\beta$-galactosidase activity was observed in arteries populated by neural crest-derived SMCs including the aorta (from the aortic root to the point of insertion of the DA), the internal carotid arteries (ICA), the proximal subclavian arteries (SC), and the DA (Figure 2B). By contrast, $\beta$-galactosidase activity was not observed in $R 26 R^{+}$control mice (Figure 2, A and B). Of note, in the ascending aorta (AAo) and aortic arch, $\beta$-galactosidase activity is observed in most but not all of SMCs populating the tunica media (Figure $2 \mathrm{C}$ ). Moreover, relatively few cells in the pulmonary artery express $\beta$-galactosidase, while almost all SMCs in the DA stained intensely blue (Figure 2D).

Wnt1-Cre-mediated recombination was observed beginning at E9.5 in neural crest-derived SMCs populating the cardiac outflow tract and branchial arch arteries (data not shown). At E11.5, 


\section{Table 1}

Genotype distribution of offspring generated from intercrossing Myocd $^{\mathrm{F} /} /$ Wnt1-Cre ${ }^{+}$mice with Myocd ${ }^{\mathrm{F} / \mathrm{F}}$ mice

$\begin{array}{lccc}\text { Genotype } & \text { E11.5-E16.5 } & \text { P2 } & \text { P21 } \\ \text { Myocd }^{+/ F} & 9 & 24 & 65 \\ \text { Myocd }^{\text {F/F }} & 10 & 22 & 63 \\ \text { Myocd }^{+/ F} / \text { Wnt1-Cre } & 11 & 22 & 66 \\ \text { Myocd }^{+/ F} / \text { Wnt1-Cre } & 10(25 \%) & 24^{+}(26 \%) & 2(1 \%)\end{array}$

AAll mice died spontaneously prior to P3.

$\beta$-galactosidase activity was observed in the outflow tract endocardial cushions as well as the proximal aorta and DA (Figure 2, $\mathrm{E}$ and $\mathrm{F}$ ). Consistent with these results, at $\mathrm{P} 2$ nuclear expression of myocardin protein (dark brown stain) was observed in medial SMCs populating the carotid artery and arch of the aorta in control mice $\left(\right.$ Myocd $\left.^{F / F}\right)$ (Figure 2, G and I), whereas little or no myocardin expression was detectable in SMCs populating the carotid artery and aorta in $\mathrm{Myocd}^{F / F} / \mathrm{Wnt1}-\mathrm{Cre}^{+}$mutant pups (Figure $2, \mathrm{H}$ and $\mathrm{J}$ ). Taken together, these data demonstrate that the conditional gene-targeting strategy employed led to very efficient excision of the Myocd gene in cardiac neural crest-derived SMCs populating the great arteries.

Conditional deletion of myocardin in neural crest-derived VSMCs results in perinatal lethality. In contrast to myocardin-null mutant embryos, which survived only to E10.5 (25), Myocd ${ }^{F / F} /$ Wnt1-Cre $^{+}$mutant pups were born in the anticipated Mendelian ratio (Table 1). At birth Myocd $^{F / F} /$ Wnt1-Cre $e^{+}$pups were indistinguishable from control littermates. However, shortly after birth Myocd $^{F / F} /$ Wnt1$\mathrm{Cr}^{+}$pups became cyanotic, and with rare exception mutant embryos died prior to P3. To confirm that neural crest-restricted deletion of myocardin was responsible for the observed phenotype, homozygous myocardin conditional mutant mice $\left(\right.$ Myocd $\left.^{F / F}\right)$ were intercrossed with Pax3-Cre transgenic mice, which also direct highly efficient Cre-mediated recombination in the neural crest (36). Homozygous conditional mutant mice (Myocd $d^{F / F} / \mathrm{Pax} 3$ $\mathrm{Cr}^{+}$) were born in the anticipated Mendelian ratio. However, once again, these conditionally targeted myocardin mutant mice died prior to P3 (data not shown).

\section{Figure 3}

MyocdF/F/Wnt1-Cre+ mutant mice exhibit PDA. (A) Intracardiac injection of toluidine blue reveals normal patterning of cardiac outflow tract and great arteries in a P2 Myocd ${ }^{F / F}$ control mouse. At P2 the DA is functionally occluded, giving rise to the ligamentum arteriosus (LA). RCA, right CA; LCA, left CA. (B) H\&E-stained transverse section cut at the level of the AAo and DAo in a P2 Myocd ${ }^{F / F}$ control mouse, demonstrating occlusion of the DA and concomitant formation of the LA. $\mathrm{Br}$, bronchus; Eso, esophagus. (C) Serial H\&E-stained section cut at the level of the PA and DAo of a P2 MyocdF/F control mouse. (D) H\&E-stained caudal section cut at the level of the PA and DAo of a P2 MyocdF/F control mouse. (E) Intra-injection of toluidine blue demonstrating widely PDA in P2 MyocdF/F/Wnt1-Cre+ mutant mouse. (F) H\&E-stained transverse section cut at the level of the AAo and DA of Myocd $^{F / F} / W_{n t 1-C r e}$ mutant mouse demonstrating widely PDA. (G) Serial H\&E-stained more caudally cut section at the level of the AAo and DA of $\mathrm{Myocd}^{\mathrm{F} / F} / \mathrm{Wnt} 1-\mathrm{Cre}^{+}$mutant mouse demonstrating widely PDA. (H) Serial H\&E-stained caudal section cut at the level of the PA and DA of $M y o c d^{F / F / W n t 1-C r e}{ }^{+}$mutant mouse demonstrating PDA. Original magnification, $\times 10$ (A and E); $\times 200$ (B-D and F-H).
Myocardin mutant mice exhibit PDA. MRTF-B mutant mice exhibit a spectrum of cardiovascular patterning defects attributable to a cell autonomous block in differentiation of the cardiac neural crest (29-31). Given the conserved structure and overlapping patterns of expression of myocardin and MRTF-B in the developing embryo (18), we postulated that Myocd ${ }^{F / F} /$ Wnt1-Cre ${ }^{+}$pups might exhibit common forms of congenital heart disease attributable to alterations in neural crest patterning of the cardiac outflow tract and great arteries. However, defects in patterning of the cardiac outflow tract and great arteries were not observed in $24 \mathrm{Myocd}^{F / F} / \mathrm{Wnt1}-\mathrm{Cre}^{+}$ mutant mice examined at P2 (Figure 3, A and E). However, intracardiac injection of toluidine blue revealed a widely PDA in all 24 Myocd $^{F / F} /$ Wnt1-Cre $e^{+}$mutants that were analyzed at P2 (Figure 3E). By contrast, the DA was occluded in each of the 22 control littermates analyzed at P2 (Figure 3A). Histological examination of the DA harvested from control and mutant mice at $\mathrm{P} 2$ revealed complete occlusion of the DA in control mice (Figure 3, B-D, arrow), but ductal patency in Myocd ${ }^{F / F} / \mathrm{Wnt1}-\mathrm{Cre}^{+}$mutant mice (Figure 3, F-H). Similarly, at P2 all $15 \mathrm{Myocd}^{F / F} / \mathrm{Pax}^{-\mathrm{Cr}^{+}}$mutant mice analyzed exhibited PDA, but patterning of the cardiac outflow tract and great arteries was not disturbed (data not shown).

Diminished expression of SMC contractile proteins in the DA of $M y o c d^{F / F}$ / Wnt1-Cre ${ }^{+}$mutant mice. Closure of the DA in the neonatal period is dependent upon functional contraction of ductal SMCs (37). Examination of the DA of E16.5 $\mathrm{Myocd}^{F / F} / \mathrm{Wnt1}^{-\mathrm{Cre}^{+} \text {mutant }}$ embryos revealed markedly diminished expression of SMC contractile proteins, including SM- $\alpha$-actin (SMA), SM-myosin heavy

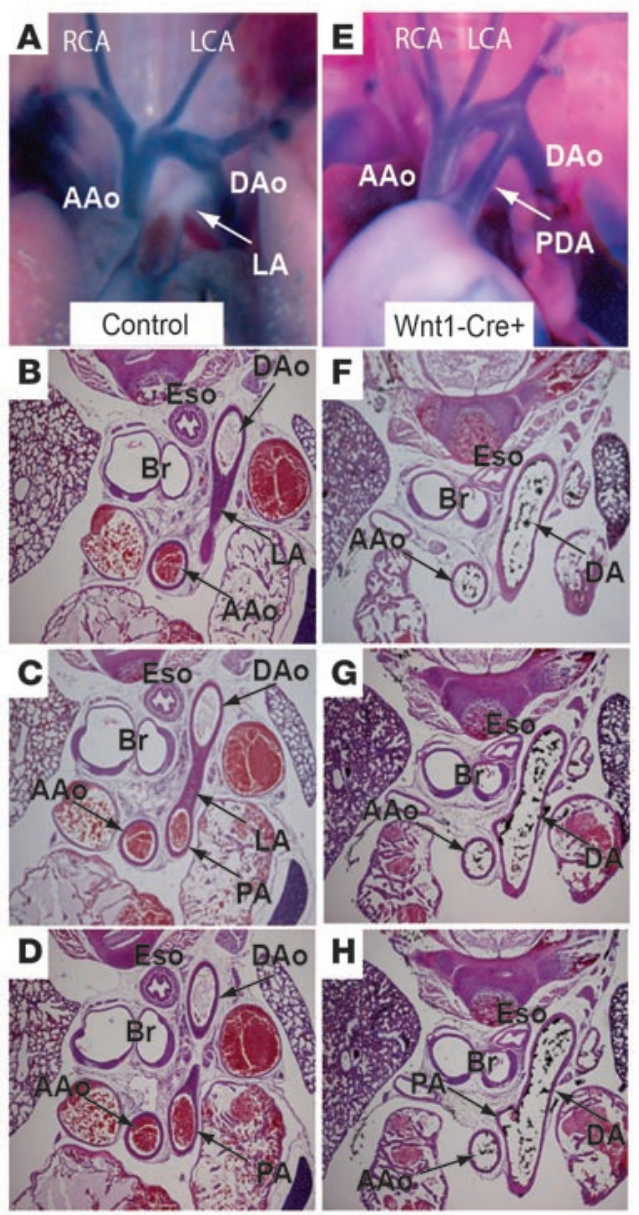




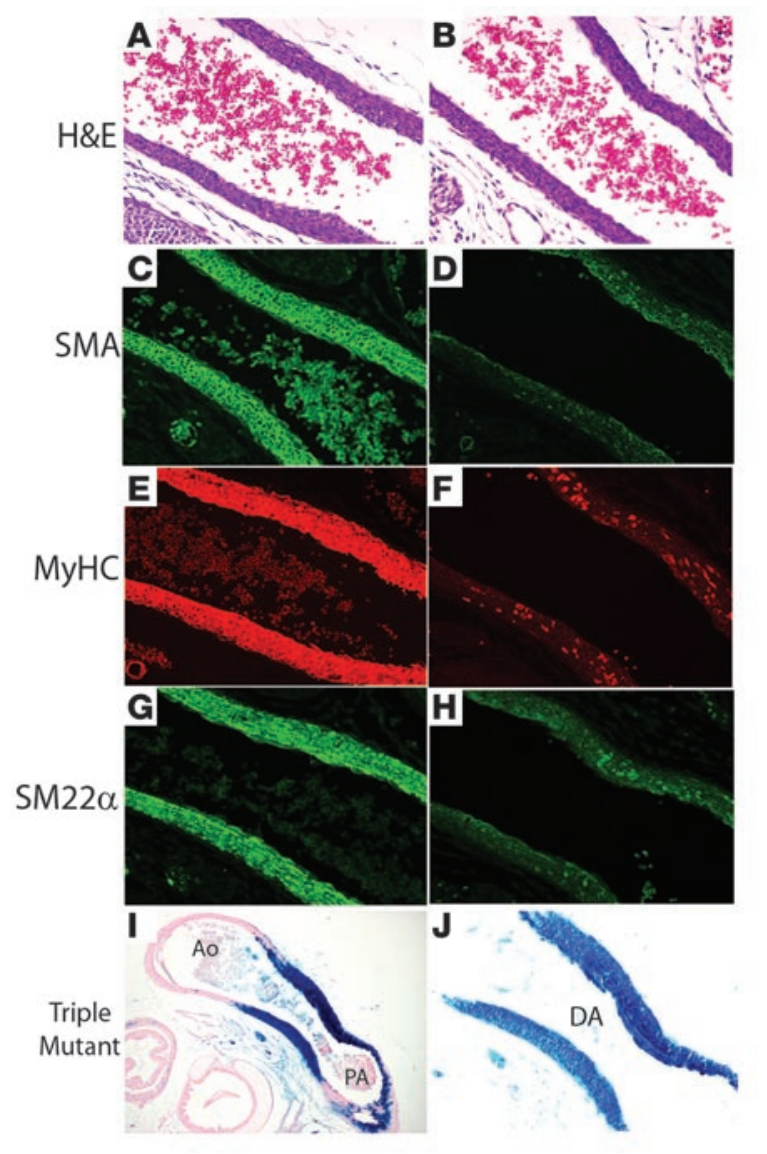

chain (SM-MyHC), and SM22 $\alpha$ in medial SMCs (Figure 4, B, D, F, and $\mathrm{H}$ ) compared with control embryos (Figure 4, A, C, E, and $\mathrm{G})$. Consistent with these findings, expression of SMC contractile proteins was markedly diminished in the DA in $\mathrm{Myocd}^{\mathrm{F} / \mathrm{F}} / \mathrm{Pax} 3-\mathrm{Cr} \mathrm{C}^{+}$ mutant mice (data not shown).

To confirm that the marked decrease in SMC contractile protein expression observed in the DA of Myocd $^{F / F} /$ Wnt1-Cre $e^{+}$mutant mice was attributable to neural crest-derived SMCs, Myocd $^{F /+} /$ Wnt1$\mathrm{Cr}^{+}$mice were bred into the $R 26 \mathrm{R}$ genetic background to generate Myocd $^{F / F} /$ Wnt1-Cre $^{+} / \mathrm{R}^{26 R^{+}}$triple mutant mice. In triple mutant mice, cells undergoing Cre-mediated recombination express $\beta$-galactosidase (recognized as blue stain). As anticipated, $M y o c d^{F / F} /$ Wnt1-Cre ${ }^{+} / \mathrm{R}^{26 \mathrm{R}^{+}}$triple mutants died prior to P3, and all triple mutants exhibited PDA, and expression of SMC contractile proteins was markedly diminished in the DA of triple mutant mice (data not shown). Remarkably, essentially all SMCs populating the DA of the triple mutant mice stained intensely blue (Figure 4, I and J) confirming that these cells are of neural crest origin and that myocardin-deficient neural crest cells migrate appropriately to the cardiac outflow tract and DA. Taken together, these data demonstrate that myocardin regulates expression of SMC contractile genes in neural crest-derived SMCs populating the DA.

Myocardin conditional mutant mice exhibit a generalized block in neural crest-derived SMC differentiation. The finding of severely diminished expression of SMC contractile proteins in the DA, raised the question of whether myocardin plays a unique role in regulating genes encoding SMC contractile proteins in the DA or whether this merely reflects a generalized defect in SMC differentiation

\section{Figure 4}

SMCs populating the DA of Myocd conditional mutant mice exhibit markedly diminished expression of SMC contractile proteins. (A and B) H\&Estained section of the DA of MyocdF/F control mouse (A) and MyocdF/F/ Wnt1-Cre ${ }^{+}$mutant mouse (B). (C, E, and G) Immunohistochemical analyses of the DA of E16.5 MyocdF/F control mouse demonstrating robust expression of SMA (green staining) (C), SM-MyHC (red staining) (E), and SM22 $\alpha$ (green staining) (G). (D, F, and H) By contrast, expression of SMA (D), SM-MyHC (F), and SM22 $\alpha(\mathbf{H})$ is markedly diminished in the DA of E16.5 MyocdF/F/Wnt1-Cre+ mutant mice. (I and J) X-gal-stained section of P2 MyocdF/F/Wnt1-Cre+/R26R+ triple mutant mice revealed that essentially all SMCs populating the DA express $\beta$-galactosidase (blue stain), confirming that these cells are of neural crest origin. Original magnification, $\times 100(\mathbf{A}-\mathbf{I}) ; \times 200(\mathbf{J})$.

in neural crest-derived SMCs. Immunohistochemical staining of the ascending aorta and aortic arch of Myocd $d^{F / F} / \mathrm{Wnt1}-\mathrm{Cr} \mathrm{C}^{+}$ mutant mice revealed markedly diminished expression of SMA, SM-MyHC, and SM22 $\alpha$ (Figure 5, B, E, H, and K) compared with their control littermates (Figure 5, A, D, G, and J). As anticipated, robust levels of SMC contractile proteins were observed in the descending aorta of Myocd ${ }^{F / F} /$ Wnt1-Cre ${ }^{+}$mutant mice, which is populated with SMCs arising from the lateral mesoderm (Figure 5, C, F, I, and L). Real-time RT-PCR performed on mRNA harvested from the proximal aorta (isolated from the aortic root to the insertion site of the DA) of 4 P2 myocardin conditional mutant mice (gray bars) and control pups (black bars) revealed $70 \%-80 \%$ decreases in expression of SMA, calponin-h1 (calponin), SM-MyHC, and SM22 $\alpha$ mRNA, respectively, in $\mathrm{Myocd}^{F / F} / \mathrm{Wnt1}-\mathrm{Cre}{ }^{+}$ mutant mice compared with control Myocd $^{F / F}$ mice (Figure $5 \mathrm{M}$ ). These data demonstrate that myocardin regulates expression of genes encoding SMC contractile proteins in neural crest-derived SMCs populating the cardiac outflow tract and great arteries.

Myocardin-mediated regulation of SMC phenotype. Electron microscopic analysis of the DA in 3 P2 Myocd $^{F / F} / W n t 1-C r e^{+}$mutant pups and 3 control littermates revealed marked disruption of arterial architecture as well as profound changes in the phenotype of SMCs in the mutant mice (Figure 6). At P2 the tunica media of the DA in control mice is composed of longitudinally and spirally arranged layers of SMCs within loose connective layers of elastic tissue (Figure 6, A and C) (38). By contrast, in Myocd ${ }^{F / F} /$ Wnt1-Cre $e^{+}$mutant mice the architecture of the neointima and tunica media was markedly disturbed (Figure 6, B and D). In the mutant DA there was a dramatic increase in ECM and a relative loss of SMC volume (Figure 6, $\mathrm{C}$ and $\mathrm{D})$. In the conditional mutant mice, SMC size was heterogeneous with a loss of spindle-like cell morphology. High-power magnification revealed relatively few myofibers (arrows) in mutant SMCs compared with control SMCs (Figure 6, E and F). Surprisingly, there was also a marked increase in synthetic organelles including the rough endoplasmic reticulum (open arrows) and Golgi in Myocd $^{F / F} /$ Wnt1-Cre ${ }^{+}$mutants compared with control littermates (Figure 6, G and H). Nuclear membrane blebbing and/or nuclear condensation, indicative of apoptosis, were not observed in ductal SMCs of the myocardin mutant mice (data not shown). Consistent with this finding, no differences were observed in TUNEL-staining in the tunica media of the DA of $\mathrm{Myocd}^{F / F} / \mathrm{Wnt1}-\mathrm{Cre}^{+}$mice and control littermates harvested from E12.5 to P2 (data not shown).

These data suggested that vascular SMCs in the DA of Myocd $d^{F / F}$ / Wnt1-Cre ${ }^{+}$mutants assume a synthetic phenotype. To determine whether ECM proteins accumulate in the DA of $\mathrm{P} 2$ myocardin con- 

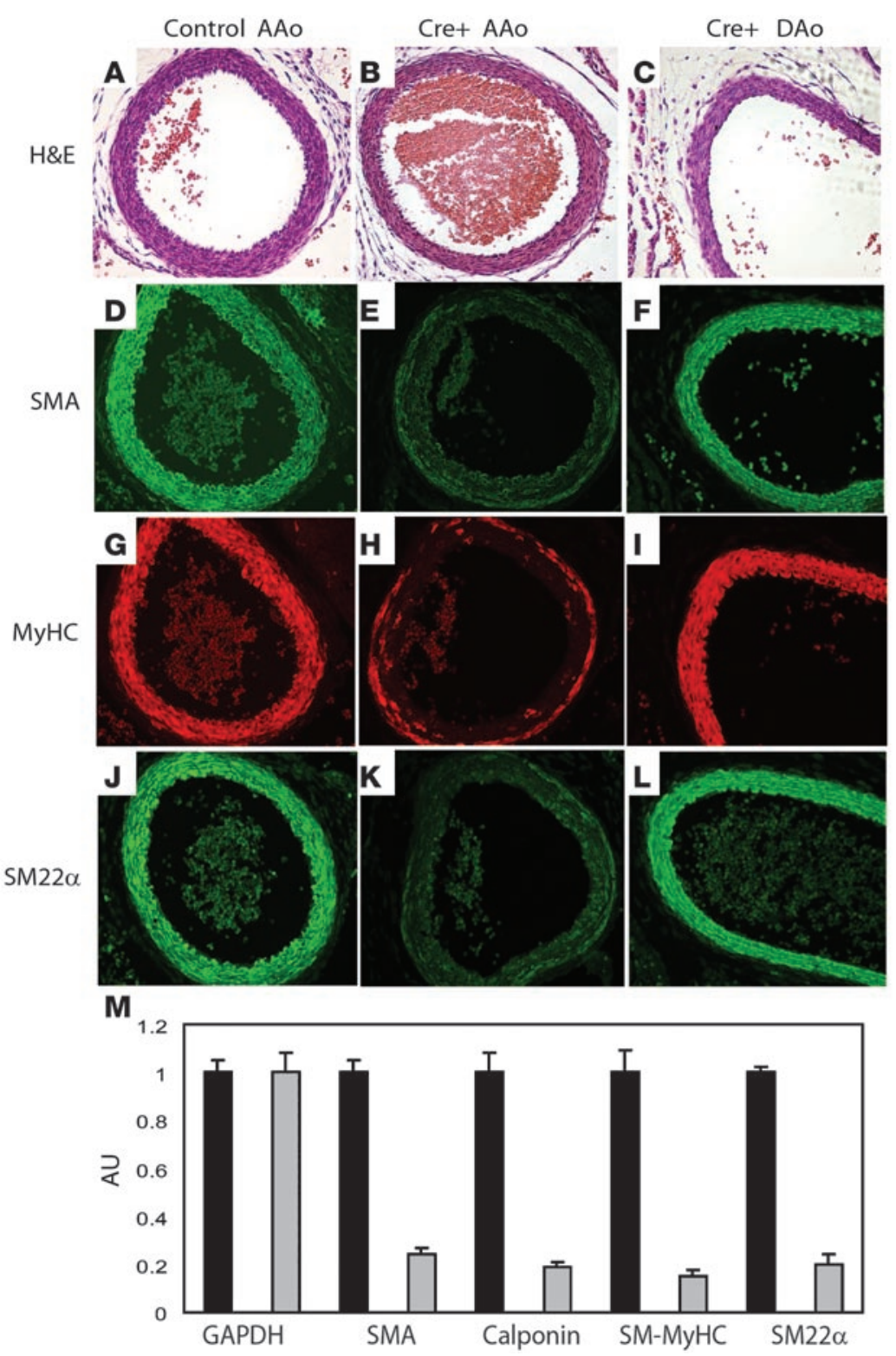

\section{Figure 5}

Myocd ${ }^{F / F} /$ Wnt1-Cre ${ }^{+}$mutant mice exhibit a generalized defect in vascular SMC differentiation. (A, D, G, and J) Serial histological sections of the AAo of an E16.5 MyocdF/F control mouse. (A) H\&E-stained AAo. (D, G, and J) Robust expression of SMA (D), SM-MyHC (G), and SM22 $\alpha(\mathbf{J})$ is observed. (B, E, H, and $\mathbf{K}$ ) Serial histological sections showing the AAo of an E16.5 Myocd ${ }^{F / F} /$ Wnt1-Cre+ mutant mouse. (B) H\&E-stained AAo. (E, H, and K) Expression of SMA (E), SM-MyHC (H), and SM22 $\alpha(\mathbf{K})$ is markedly diminished in the AAo of $\mathrm{Myocd}^{\mathrm{F} / \mathrm{F} / W n t 1-\mathrm{Cre}^{+}}$ mutant mouse. (C, F, I, and L) Serial histological

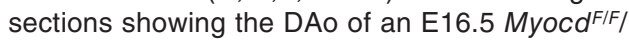
Wnt1-Cre ${ }^{+}$mutant mouse. (C) H\&E-stained section of the DAo. $(\mathbf{F}, \mathbf{I}$, and $\mathbf{L})$ Robust expression of SMA $(\mathbf{F})$, SM-MyHC (I), and SM22 $\alpha(\mathbf{L})$ is observed in the DAo of a MyocdF/F/Wnt1-Cre ${ }^{+}$mutant mouse. Original magnification, $\times 200$. (M) RT-PCR analysis of SMC contractile gene expression in MyocdF/F/ Wnt1-Cre ${ }^{+}$mutant and control mice. mRNA harvested from the proximal aorta, carotid arteries, and DA of 4 P2 Myocd $/ F / F / W n t 1-C r e^{+}$mice and 4 control mice was analyzed for expression of GAPDH, SMA, calponin-h1 (calponin), SM-MyHC, and SM22 $\alpha$ by real-time RT-PCR as described (28). Data are expressed as mean gene expression (arbitrary units) \pm SEM. Gray bars represent gene expression

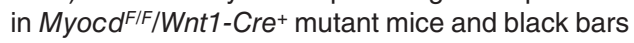
represent gene expression in control mice. ditional mutant mice, sections of the DA were stained with antibodies that recognize fibronectin and laminin and visualized by confocal microscopy (Figure 7). A dramatic increase in fibronectin expression was observed in the DA of $\mathrm{Myocd}^{\mathrm{F} / \mathrm{F}} / \mathrm{Wnt1}$-Cre $\mathrm{C}^{+}$mutants compared with control mice (compare Figure 7, A and B). In addition, a marked increase in expression of laminin was observed in the DA of myocardin conditional mutant mice (Figure 7, C and D). Taken together, these data demonstrate that myocardin regulates expression of contractile genes required for closure of the DA. Moreover, these data reveal that myocardin-deficient SMCs assume a synthetic phenotype manifested by changes in cellular morphology and a concomitant increase in synthetic organelles and ECM.

$M y o c a r d i n$ regulates maintenance of the SMC contractile phenotype. To determine whether myocardin is required for maintenance of the differentiated SMC phenotype, primary cultures of mouse aortic SMCs harvested from myocardin conditional mutant mice $\left(\right.$ Myocd $\left.^{\mathrm{F} / F}\right)$ were infected with Ad-Cre, a replication-defective adenovirus
(RdAV) encoding Cre recombinase, or the control virus Ad-GFP, which encodes green fluorescent protein (GFP). The transduction efficiency of SMCs following RdAV infection was greater than $90 \%$ under the experimental conditions employed. Consistent with this finding, RT-PCR confirmed that myocardin mRNA decreased by $90 \%$ in SMC cultures infected with Ad-Cre relative to levels of myocardin gene expression observed in Ad-GFP-infected cells. Six days after infection, transduced SMCs were harvested and stained with antibodies that recognize Cre recombinase, SM-MyHC, calponin-h1, SM22 $\alpha$, and SMA, respectively. In aortic SMCs infected with the control virus Ad-GFP, colocalization of green staining with red staining, indicative of SM-MyHC expression, was observed (Figure 8, A-C). By contrast, expression of SM-MyHC (red staining) was not observed in cells transduced with Ad-Cre (green stain) (Figure 8, D-F). Similarly, expression of calponin-h1 (compare Figure 8, G-I with Figure 8, J-L), SM22 $\alpha$ (compare Figure 8, M-O with Figure 8, P-R), and SMA (data not shown) was not observed in Myocd ${ }^{F / F}$ SMCs transduced with the 


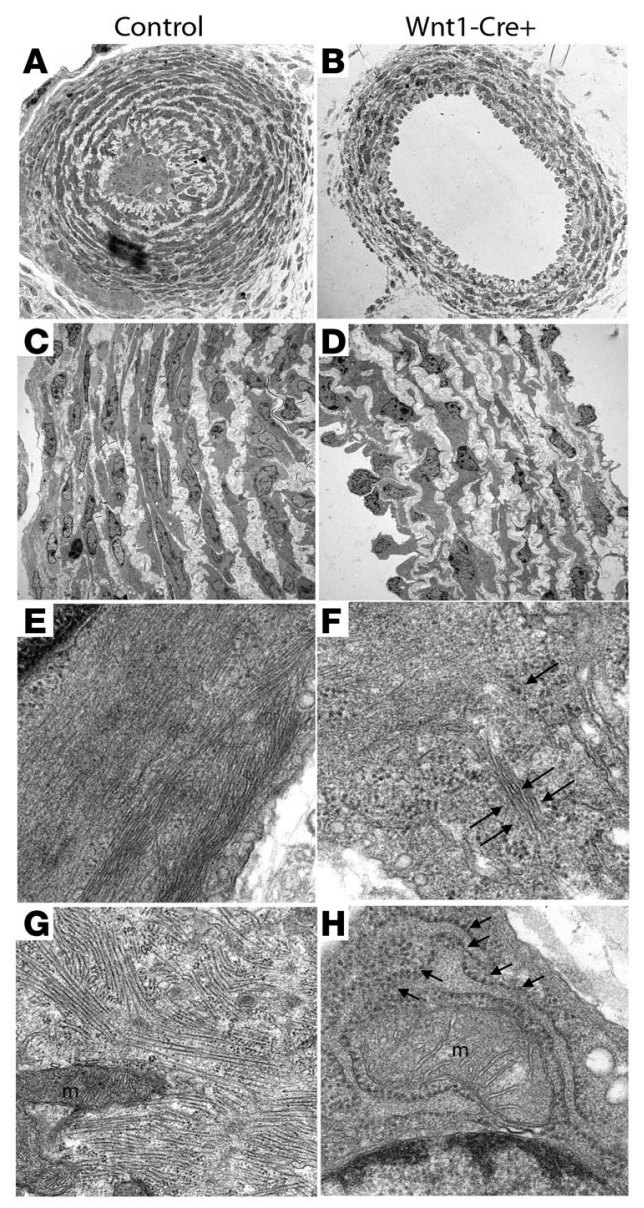

Ad-Cre virus. Of note, $M y o c d^{F / F}$ SMCs transduced with the Ad-Cre were easily distinguished from nontransduced SMCs by their appearance, which was smaller and more polygonal.

To quantify expression of proteins in Myocd $^{F / F}$ SMCs transduced with Ad-Cre and Ad-GFP, respectively, Western blot analyses were performed with whole cell lysates prepared from the transduced SMCs. As anticipated, high levels of GFP were observed in cells transduced with Ad-GFP, while abundant Cre recombinase was detected in cells infected with Ad-Cre (Figure 8S). Consistent with the immunohistochemical analyses, expression of contractile proteins including SMA and SM22 $\alpha$ was dramatically decreased in Myocd $^{F / F}$ SMCs transduced with Ad-Cre relative to levels observed in cells transduced with Ad-GFP. By contrast, expression of GAPDH was comparable in SMCs transduced with Ad-Cre and Ad-GFP. Taken together, these data demonstrate that myocardin is required for maintenance of contractile protein expression in vascular SMCs. Of note, the primary aortic SMCs utilized in these experiments are derived from the cardiac neural crest and lateral mesoderm.

\section{Discussion}

Transcriptional coactivators provide a flexible mechanism to expand information encoded within the genome in response to developmental and environmental stimuli (reviewed in ref. 18). Myocardin is a potent transcriptional coactivator expressed exclusively in cardiomyocytes and SMCs (22). Myocardin physically associates with the MADS box transcription factor SRF, activating expression of a set of genes encoding SMC contractile pro-

\section{Figure 6}

Ultrastructural changes in vascular SMCs populating the DA of Myocd $^{F / F} /$ Wnt1-Cre ${ }^{+}$mutant mice. The DA of P2 MyocdF/F control (A, $\mathbf{C}, \mathbf{E}$, and $\mathbf{G})$ and Myocd ${ }^{F / F} /$ Wnt1-Cre+ ${ }^{+}$utant $(\mathbf{B}, \mathbf{D}, \mathbf{F}$, and $\mathbf{H})$ mice $(n=3$ of each) were isolated, fixed, stained, and analyzed by electron microscopic analyses. (A and B) Low-power view demonstrating luminal occlusion of the DA in a control mouse (A), but widely PDA in the MyocdF/F/Wnt1-Cre+ mutant mouse (E). Original magnification, $\times 500$. (C) The tunica media of the DA in control mice exhibits regular circumferential organization with typical spindle-like morphology of SMCs. (D) By contrast, there is a loss of SMC mass with marked variability in SMC size and morphology as well as a concomitant increase in ECM in the mutant DA. Original magnification, $\times 25,000$. (E) SMC morphology and ultrastructure in control Myocd ${ }^{F / F}$ DA demonstrating abundant myofibrils. Original magnification, $\times 25,000$. (F) SMC morphology and

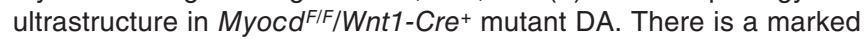
decrease in myofibrils (large arrows) and a concomitant increase in synthetic organelles including rough endoplasmic reticulum (small arrows). Original magnification, $\times 100,000$. (G and $\mathbf{H}$ ) Comparison of SMC morphology in control $(\mathbf{G})$ and mutant $(\mathbf{H})$ DA demonstrating a relative increase in organelles associated with synthetic functions, including rough endoplasmic reticulum (arrows) and vacuoles (V) in MyocdF/F/Wnt1-Cre+ mutant SMC (H) compared with the control SMC (G). m, mitochondria; n, nucleus. Original magnification, $\times 100,000$.

teins (reviewed in refs. 18, 19). To determine the cell autonomous function(s) of myocardin in cardiac neural crest-derived SMCs that populate the cardiac outflow tract and great arteries, we generated and characterized mice in which the myocardin gene was selectively ablated in neural crest-derived SMCs. Myocd ${ }^{F / F} / \mathrm{Wnt}^{-C}-\mathrm{Cr}^{+}$and Myocd $^{F / F} / \mathrm{Pax}_{3}-\mathrm{Cre}^{+}$mice survive to birth, but die prior to P3 from complications related to PDA. A dramatic reduction in contractile proteins was observed in neural crest-derived SMCs populating the DA and great arteries of Myocd conditional mutant mice. Surprisingly, Myocd-deficient vascular SMCs also exhibited features associated with the synthetic SMC phenotype. Moreover, ablation of the Myocd gene in primary aortic SMCs harvested from conditionally gene-targeted mice led to a profound decrease in contractile SMC protein expression. Taken together, these data provide unequivocal evidence that myocardin regulates expression of genes encoding SMC proteins required for maintenance of the contractile SMC phenotype. In addition, these data provide new insights into the molecular and genetic programs underlying PDA.

Myocardin-null mutant mice survive only to E10.5, exhibiting defects in the embryonic and yolk sac vasculature, growth retardation, and developmental delay (25). By contrast, MyocdF/F/Wnt1$\mathrm{Cre}^{+}$and $\mathrm{Myocd}^{F / F} / \mathrm{Pax} 3-\mathrm{Cre}^{+}$mice survived to birth but died prior to $\mathrm{P} 3$ from complications related to PDA. What might account for these distinct phenotypes? As with most mice generated using conditional gene-targeting strategies, the phenotype of Myocd $^{F / F} /$ Wnt1$\mathrm{Cre}^{+}$and $\mathrm{Myocd}^{\mathrm{F} / \mathrm{F}} / \mathrm{Pax} 3-\mathrm{Cre}^{+}$mutant mice represents a hypomorphic, as opposed to true null, phenotype. Low levels of myocardin expression were detectable in some SMCs populating the cardiac outflow tract, aorta, and great arteries (Figures 4 and 5). Moreover, cell fate mapping studies have shown that some SMCs populating the ascending aorta are derived from the secondary or anterior heart field (39). As such, these cells may play an important role in cardiac outflow tract patterning. However, we believe that the observed differences between Myocd-null embryos and Myocd ${ }^{F / F}$ / Wnt1-Cre ${ }^{+}$and Myocd $^{F / F} / \mathrm{Pax}_{3}-\mathrm{Cre}^{+}$pups is principally related to the neural crest-restricted conditional gene-targeting strategy employed in these experiments. Both the Wnt 1 and Pax 3 promoters 


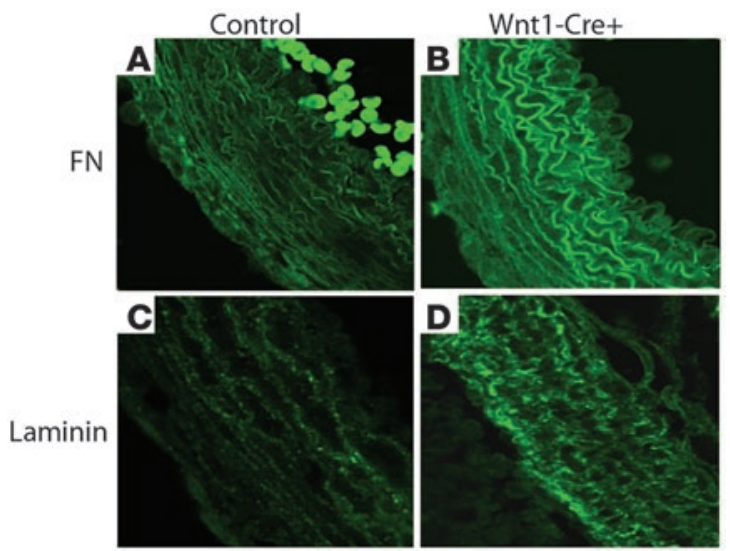

efficiently target neural crest-derived SMCs populating the cardiac outflow tract and great arteries (34). SMCs populating the extraembryonic or yolk sac vasculature are not neural crest-derived. As such, the Wnt1-Cre and Pax3-Cre gene-targeting lines utilized in these studies serve to distinguish the function(s) of myocardin in the embryonic vasculature from its function(s) in the extra-embryonic vasculature. Consistent with this, we have recently observed profound defects in the yolk sac vasculature and evidence of generalized intrauterine growth retardation in $\mathrm{CMV}-\mathrm{Cre}^{+} / \mathrm{Myocd}^{F / F}$ mutant embryos (J. Huang and M. Parmacek, unpublished observations). In addition, Olson and colleagues have reported defects in the yolk sac vasculature of myocardin-null embryos (19).

The capacity of vascular SMCs to modulate their phenotype in response to vascular injury has been implicated in the pathogenesis of vascular proliferative syndromes, including atherosclerosis and restenosis following balloon angioplasty and/or intravascular stenting (reviewed in ref. 40). We and others have shown that myocardin functions as a transcriptional coactivator, directly activating transcription of genes encoding SMC contractile proteins (reviewed in ref. 18). Therefore, it was not surprising that in myocardin conditional mutant mice expression of SMC-restricted contractile proteins was dramatically downregulated in the DA and neural crest-derived great arteries. By contrast, the demonstration that myocardin-deficient SMCs in the intact vasculature assume a synthetic SMC phenotype was not anticipated (reviewed in ref. 40). These data place myocardin at a critical nodal point required for maintenance of the contractile SMC phenotype. However, it remains unclear whether myocardin directly and/or indirectly suppresses the synthetic SMC phenotype. At a molecular level, these processes may be functionally coupled, at least in part, via direct competition for binding to SRF between myocardin and ternary complex factors (TCFs) in the Ets family (41).

It remains unclear what mechanisms distinguish the functions of MRTFs in tissues where they are coexpressed. Genetically engineered mice harboring mutations in myocardin, MRTF- $A$ and $M R T F-B$, respectively, exhibit distinct phenotypes (18). Disruption of MRTF-B gene results in profound patterning defects of the cardiac outflow tract and great arteries recapitulating common forms of congenital heart disease (29-31). Like the myocardin conditional mutant mice, the MRTF- $B$ mutants demonstrated a marked decrease in expression of genes encoding SMC contractile proteins (29-31). However, in contrast to the myocardin conditional mutants, MRTF-B mutant mice exhibit abnormal patterning of the branchial arch arteries as early as E11.0-11.5. In this regard it is noteworthy that MRTF-B is expressed as early as E8.0 in car-

\section{Figure 7}

Accumulation of ECM protein in the DA of P2 MyocdF/F/Wnt1-Cre+ mutant mice. Sections of the DA were stained with antibodies that recognize fibronectin (FN) and laminin and visualized by confocal microscopy. A dramatic increase in fibronectin expression was observed in

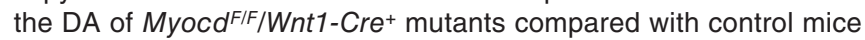
(compare $\mathbf{A}$ and $\mathbf{B}$ ). In addition, a marked increase in expression of laminin was observed in the DA of myocardin conditional mutant mice (compare $\mathbf{C}$ and D). Original magnification, $\times 200$.

diac neural crest cells populating rhombomeres 3 and 5 (29), while myocardin mRNA is not detected in the hindbrain of the unturned mouse embryo (J. Li and M. Parmacek, unpublished observations). Therefore, a developmental window exists between E8.0 and E9.5 when MRTF-B is expressed in the cardiac neural crest cells and myocardin is not. We postulate that expression of MRTF-B in cardiac neural crest cells during this critical window of time provides an instructive signal required for proper patterning of the branchial arch arteries that impacts on patterning of the aorta and great arteries. Alternatively, myocardin and MRTF-B may differentially respond to intracellular signals and/or activate distinct subsets of target genes. The precise molecular targets of MRTF-B and myocardin in the cardiac neural crest and neural crest-derived SMCs are the subject of ongoing investigation.

The demonstration that Myocd ${ }^{F / F} /$ Wnt1-Cre ${ }^{+}$and Myocd $^{F / F} / \mathrm{Pax} 3-\mathrm{Cr} e^{+}$ pups succumb to PDA in the perinatal period underscores the importance of the PDA in redirecting the fetal circulation required for postnatal survival. The DA has evolved unique anatomic and physiological properties required to regulate and direct the embryonic and postnatal circulations. The tunica media of the aorta and pulmonary artery are composed of circumferentially arranged layers of SMCs and elastic fibers. By contrast, the media of the DA is composed of longitudinally and spirally arranged layers of SMCs and a rich network of ECM and elastic fibers. After birth an increase in oxygen tension and decline in circulating PGE2 stimulates contraction of ductal SMCs required for functional separation of the pulmonary and systemic circulation (14). As such, the observed PDA in myocardin mutant mice resulted from failure of ductal SMCs to effectively contract due to marked downregulation of SMC contractile proteins. Analysis of Myocd ${ }^{F / F} / \mathrm{Wnt1}-\mathrm{Cre}^{+} / \mathrm{R}^{2} 6 \mathrm{R}^{+}$triple mutant mice confirmed that virtually all SMCs populating the mutant DA are neural crest-derived, precluding the possibility of compensation from nonneural crest-derived SMCs in the DA. Of note, neural crest-derived cells also contribute to the aortic and pulmonary valves (AV and PV). However, the AV and PV valve of P2 Myocd $d^{F / F}$ / Wnt1-Cre $e^{+} / R 26 R^{+}$triple mutants appear morphologically normal, suggesting strongly that expression of myocardin in cardiac neural crest-derived cells is not required for embryonic development of the AV or PV (J. Huang and M. Parmacek, unpublished observations).

Increasing evidence suggests that multiple genes may predispose to PDA, possibly exacerbated by environmental triggers $(7,8,11,42$, 43). The demonstration that selective ablation of Myocd in cardiac neural crest-derived SMCs populating the DA gives rise to PDA suggests myocardin and/or myocardin-regulated genes may be involved in the pathogenesis of PDA. Consistent with this hypothesis, French and American kindreds have recently been described with heritable PDA and thoracic aortic aneurysm (11). This syndrome is attributable to mutations in the smooth muscle myosin heavy chain gene (MYH11), a direct transcriptional target of myocardin (11). Because myocardin-deficient mice with PDAs did not survive beyond P3, we 


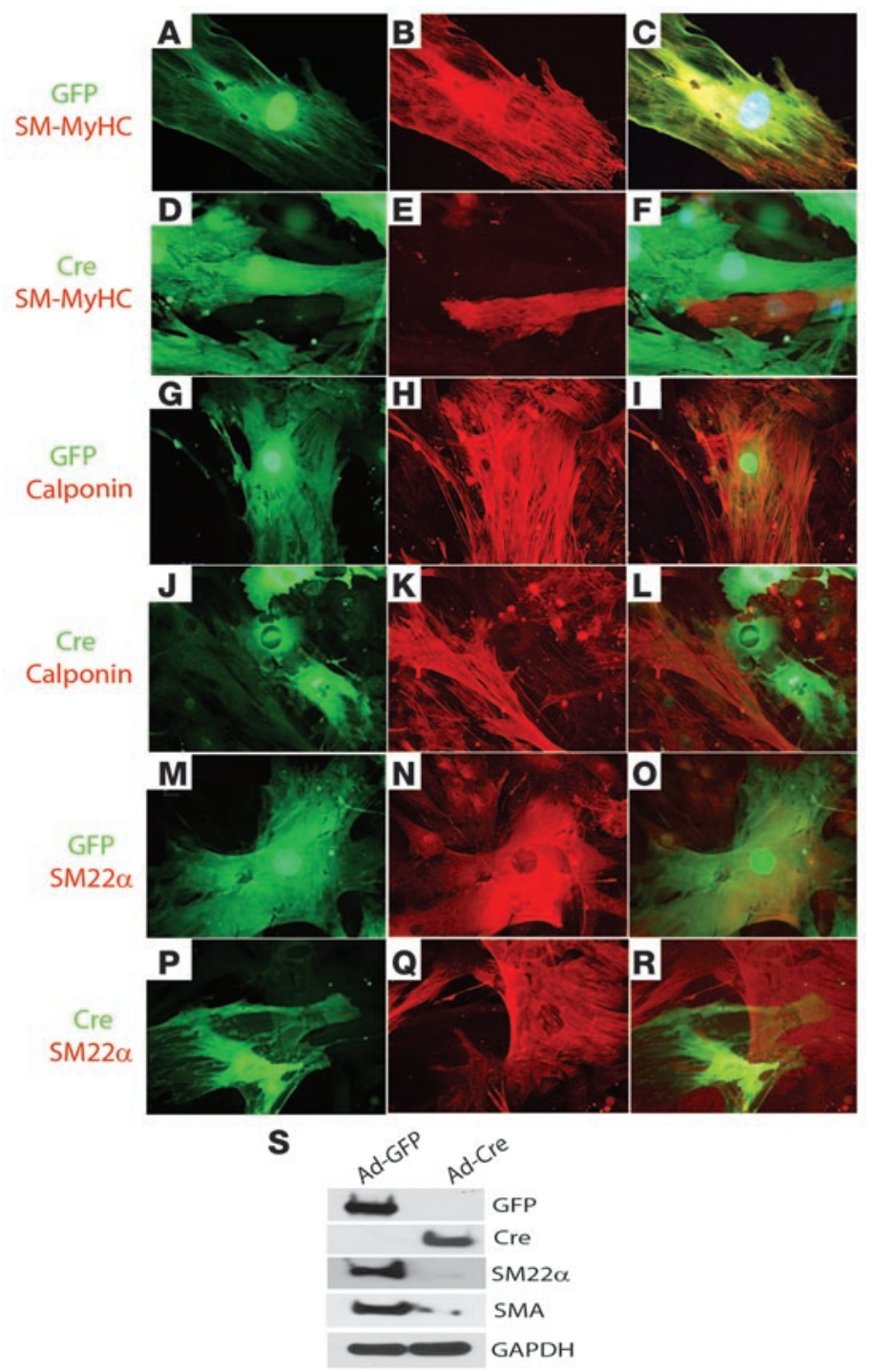

\section{Figure 8}

Myocardin-regulated maintenance of the contractile SMC phenotype. Primary SMCs isolated from MyocdF/F aortae were infected with Ad-GFP (GFP) (A-C, G-I, and $\mathbf{M}-\mathbf{O})$ or Ad-Cre recombinase (Cre) (D-F, J-L, and $\mathbf{P}-\mathbf{R})$. Six days after infection, SMCs were immunostained with antibodies that recognize GFP, Cre, SMMyHC (B, C, E, and F), calponin-h1 (H, I, K, and L), and SM22 $\alpha$ $(\mathbf{N}, \mathbf{O}, \mathbf{Q}$, and $\mathbf{R})$, respectively. SMCs transduced with GFP and Cre stain green, while SMC contractile proteins stains red. (A-C) Colocalization of GFP and MyHC is observed. (D-F) By contrast, SMCs transduced with Ad-Cre (green) do not stain red indicating decreased SM-MyHC expression. (G-I) Colocalization of GFP and calponin is observed. ( $\mathbf{J}-\mathbf{L})$ SMCs transduced with Ad-Cre do not stain red indicating decreased calponin expression. (M-O) Colocalization of GFP and SM22 is observed. SMCs transduced with Ad-Cre do not stain red indicating decreased SM22 $\alpha$ expression. Original magnification, $\times 200$. (S) Western blot analysis of cell lysates harvested from MyocdF/F SMCs transduced with Ad-Cre and Ad-GFP. Abundant GFP and Cre are observed in cells transduced with Ad-GFP and Ad-Cre, respectively (rows 1 and 2). Expression of SMA and SM22 $\alpha$ was dramatically decreased in $M y o c d^{F / F}$ SMCs transduced with Ad-Cre relative to levels observed in cells transduced with Ad-GFP (lanes 3 and 4). Expression of GAPDH was comparable in SMCs transduced with Ad-Cre and Ad-GFP (lane 5). cannot determine whether over time they would develop thoracic aortic aneurysms and/or aortic dissections. However, the anatomic derangements observed with the mutant DA recapitulate many features observed in aortae of patients harboring MYH11 mutations. In any case, these data serve to identify Myocd and myocardin-regulated genes as candidate genes that may play a role in the pathogenesis of PDA and possibly diseases of the aorta.

\section{Methods}

Generation and characterization of mice with a conditional-targeted myocardin allele. The conditional gene-targeting vector was constructed via recombineering with a BAC as described (44). A BAC containing the Myocd gene (clone no. RP22-139L16) was isolated from the ARPCI-22 129 mouse BAC library. The homology arms used for subcloning and gene-targeting were PCR amplified from BAC DNA. Each arm was subcloned into the MC1TK-containing plasmid PL253 to generate a gap-repair plasmid. A mini-targeting vector was constructed and cloned into PL452 containing a floxed neomycin (Neo) selection cassette. The Neo cassette was excised with Cre recombinase, leaving a single loxP site at the targeted locus. An EcoRI restriction site was included in the mini-targeting vector to identify correctly targeted ES cells
(Figure 1A). To assess whether the loxP and FRT sites remained intact in the targeting vector, the Myocd conditional gene-targeting vector plasmid DNA was transformed into arabinose-induced EL350 and EL250 cells, respectively. Targeted cells containing the plasmid were selected with ampicillin. Southern blot analyses were performed as described previously (45). The Myocd conditional gene-targeting vector was linearized with NotI and electroporated into SV129-derived ES cells as described previously (45). Transformants were selected in G418 and ganciclovir as described (45). DNA from G418-resistant ES cell clones was analyzed by Southern blot analysis after EcoRI digestion with a radiolabeled probe derived from genomic sequences located $5^{\prime}$ of the targeting vector (Figure 1A, black rectangle). The conditionally targeted allele generates an 11.4-kb hybridization signal (wildtype) and 9.4-kb (mutant) EcoRI fragment using a 5' probe. One hundred and twenty colonies were obtained, and six colonies with the conditionally targeted Myocd allele were identified.

ES cell clones with selective Neo deletion were identified by Southern blot analysis (Figure 1, A and B). Correctly targeted cells were microinjected into C57BL/ 6 donor blastocysts as described previously (46). The resulting male chimeras were mated with C57BL/6 females, and agouti offspring were genotyped by Southern blot analysis using EcoRI-digested DNA (Figure 1A). 
Heterozygous $\mathrm{Myocd}^{+/ F}$ mice were interbred to generate homozygous conditionally targeted myocardin mice $\left(\right.$ Myocd $\left.^{F / F}\right)$ in a mixed SV129/BL6 genetic background. Genotype was determined by Southern blotting or by PCR using primers A ( $5^{\prime}$-TAAAAGAGGCTCTCCTGCTTAGGC- $\left.3^{\prime}\right)$ and B (5'-AGGTGGTTCAAAGGAAGAGCATGGGC-3'); CRE mice (Cre800F 5'GAACCTGATGGACATGTTCAGGGA-3'; Cre800R 5'-CAGAGTCATCCTTAGCGCCGTAAA-3') (Figure 1C).

Transgenic and genetically targeted mice. To delete myocardin throughout the mouse embryo, Myocd ${ }^{F / F}$ mice were interbred with CMV-Cre transgenic mice described previously (47). To delete myocardin selectively in neural crest derivatives, $M y o c d^{F / F}$ mice were interbred with Wnt1-Cre transgenic mice kindly provided by A. McMahon (Harvard University, Boston, Massachusetts, USA) (48) and Pax3-Cre transgenic mice described previously (36). Matings were performed between male compound heterozygous Myocd ${ }^{+/ F} / \mathrm{Wnt1}_{-} \mathrm{Cre}^{+}$mice or $\mathrm{Myocd}^{+/ F} / \mathrm{Pax3}-\mathrm{Cre}^{+}$ and female $\mathrm{Myocd}^{F / F}$ mice. To generate $\mathrm{Myocd}^{F / F} / \mathrm{Wnt1}-\mathrm{Cre}^{+} / \mathrm{R}^{26 \mathrm{R}^{+}}$triple mutant mice, Myocd $^{+/ F} / \mathrm{Wnt}_{1}-\mathrm{Cre}^{+}$were interbred with R26R Rosa indicator mice described previously (35). All animal experimentation was performed under protocols approved by the University of Pennsylvania IACUC and in accordance with NIH guidelines.

Immunohistochemistry and confocal microscopy. Immunohistochemistry and confocal microscopy protocols are available at http://www.med. upenn.edu/mcrc/histology_core/. Primary antibodies utilized for immunohistochemistry studies included the following: monoclonal anti-calponin antibody (hCP) (C2687), monoclonal anti-Cre antibody (clone7-23), monoclonal anti-SMA (1A4) (Sigma-Aldrich), polyclonal anti-SM22 $\alpha$ antibody (Ab10135) (Abcam); anti-smooth muscle myosin heavy chain IgG (BT-562) (Biomedical Technologies), polyclonal anti-fibronectin (Cell Signaling), monoclonal anti-GFP (Chemicon), monoclonal anti-GAPDH (Chemicon), and polyclonal anti-laminin (Sigma-Aldrich). Secondary antibodies utilized included the following: Alexa Fluor 488 goat anti-mouse and rabbit anti-mouse, Alexa Fluor 568 goat anti-rabbit and rabbit anti-mouse (Invitrogen), and donkey anti-goat IgG, donkey anti-rabbit IgG, and donkey anti-mouse IgG HRP (Jackson ImmunoResearch). Tissue sections were fixed in paraformaldehyde, and where indicated stained for $\beta$-galactosidase activity as previously described (46). TUNEL staining of $\mathrm{Myocd}^{\mathrm{F} / F} / \mathrm{Wnt1}-\mathrm{Cr} \mathrm{e}^{+}$ and Myocd $^{F / F}$ control embryos harvested at E12.5, E14.5, E16.5, and P2 was performed as described (49). Confocal microscopy was performed on a Leica PCS SP2 microscope.

Electron microscopy. Tissues were fixed in $2 \%$ glutaraldehyde with $0.1 \mathrm{M}$ sodium cacodylate ( $\mathrm{pH} 7.4$ ) for 72 hours at $4^{\circ} \mathrm{C}$. Samples were then incubated with $2 \%$ osmium tetroxide and $0.1 \mathrm{M}$ sodium cacoylate ( $\mathrm{pH} 7.4$ ) for 1 hour at $4^{\circ} \mathrm{C}$. Ultrathin sections were stained with lead citrate and uranyl acetate and were viewed on a JEM 1010 microscope (JOEL).

Infection of primary mouse aortic SMCs with replication-defective adenovirus. Primary SMCs were isolated from the aortae of 4-week-old Myocd ${ }^{F / F}$ mice using the aortic explant technique (50) and were infected (200 MOI) with Ad-GFP or Ad-Cre (University of Pennsylvania Adenoviral Vector Core). After 6 days total RNA was isolated with TRIzoL (Invitrogen Corp.), and cDNA was prepared using the SuperScript RT kit (Invitrogen Corp.). Realtime RT-PCR of SMC-restricted gene expression was performed using the DNA Engine Opticon 2 Real-Time Detection System (Bio-Rad Laboratories Inc.) as described (28). Cell lysates were prepared and Western blotting analyses performed as described previously (51).

Intracardiac toluidine blue injections. Toluidine blue was injected intracardially or into the descending aorta of mouse embryos using a pulled glass pipette and immediately photographed as described previously (29).

\section{Acknowledgments}

We thank Xiaobing Jiang and Neelima Shah for technical assistance and helpful discussions. We thank Lisa Gottschalk for assistance with figure preparation. This research was supported by NIH PO-1-HL075380 to J.A. Epstein and M.S. Parmacek, NIH K08-HL079072 to A. Proweller, and from an allocation from the Commonwealth of Pennsylvania.

Received for publication July 18, 2007, and accepted in revised form November 7, 2007.

Address correspondence to: Michael S. Parmacek, University of Pennsylvania, Cardiovascular Institute, 9035 Gates Pavilion, 3400 Spruce Street, Philadelphia, Pennsylvania 19104, USA. Phone: (215) 662-3140; Fax: (215) 349-8017; E-mail: michael.parmacek@ uphs.upenn.edu.
1. Mitchell, S.C., Korones, S.B., and Berendes, H.W. 1971. Congenital heart disease in 56,109 births. Incidence and natural history. Circulation. 43:323-332

2. McMurphy, D.M., Heymann, M.A., Rudolph, A.M., and Melmon, K.L. 1972. Developmental changes in constriction of the ductus arteriosus: responses to oxygen and vasoactive agents in the isolated ductus arteriosus of the fetal lamb. Pediatr. Res. 6:231-238.

3. Gibson, S., and Lewis, K.C. 1952. Congenital heart disease following maternal rubella. Proc. Inst. Med. Chic. 19:149.

4. Gittenberger-de Groot, A.C., Moulaert, A.J., and Hitchcock, J.F. 1980. Histology of the persistent ductus arteriosus in cases of congenital rubella. Circulation. 62:183-186.

5. Jenkins, K.J., et al. 2007. Noninherited risk factors and congenital cardiovascular defects: current knowledge: a scientific statement from the American Heart Association Council on Cardiovascular Disease in the Young: endorsed by the American Academy of Pediatrics. Circulation. 115:2995-3014.

6. Gittenberger-de Groot, A.C. 1977. Persistent ductus arteriosus: most probably a primary congenital malformation. Br. Heart J. 39:610-618.

7. Mani, A., et al. 2002. Finding genetic contributions to sporadic disease: a recessive locus at $12 \mathrm{q} 24 \mathrm{com}$ monly contributes to patent ductus arteriosus.
Proc. Natl. Acad. Sci. U. S. A. 99:15054-15059.

8. Mani, A., et al. 2005. Syndromic patent ductus arteriosus: evidence for haploinsufficient TFAP2B mutations and identification of a linked sleep disorder. Proc. Natl. Acad. Sci. U. S. A. 102:2975-2979.

9. Satoda, M., et al. 2000. Mutations in TFAP2B cause Char syndrome, a familial form of patent ductus arteriosus. Nat. Genet. 25:42-46.

10. Zhao, F., et al. 2001. Novel TFAP2B mutations that cause Char syndrome provide a genotype-phenotype correlation. Am. J. Hum. Genet. 69:695-703.

11. Zhu, L., et al. 2006. Mutations in myosin heavy chain 11 cause a syndrome associating thoracic aortic aneurysm/aortic dissection and patent ductus arteriosus. Nat. Genet. 38:343-349.

12. Smith, G.C. 1998. The pharmacology of the ductus arteriosus. Pharmacol. Rev. 50:35-58.

13. Desligneres, S., and Larroche, J.C. 1970. Ductus arteriosus. I. Anatomical and histological study of its development during the second half of gestation and its closure after birth. II. Histological study of a few cases of patent ductus arteriosus in infancy. Biol. Neonate. 16:278-296.

14. Michelakis, E., et al. 2000. Voltage-gated potassium channels in human ductus arteriosus. Lancet. 356:134-137.

15. Kovalcik, V. 1963. The response of the isolated ductus arteriosus to oxygen and anoxia. J. Physiol. 169:185-197.
16. Clyman, R.I., Heymann, M.A., and Rudolph, A.M. 1977. Ductus arteriosus responses to prostaglandin E1 at high and low oxygen concentrations. Prostaglandins. 13:219-223.

17. Heymann, M.A., Rudolph, A.M., and Silverman, N.H. 1976. Closure of the ductus arteriosus in premature infants by inhibition of prostaglandin synthesis. N. Engl. J. Med. 295:530-533.

18. Parmacek, M.S. 2007. Myocardin-related transcription factors: critical coactivators regulating cardiovascular development and adaptation. Circ. Res. 100:633-644.

19. Pipes, G.C., Creemers, E.E., and Olson, E.N. 2006. The myocardin family of transcriptional coactivators: versatile regulators of cell growth, migration, and myogenesis. Genes Dev. 20:1545-1556.

20. Creemers, E.E., Sutherland, L.B., Oh, J., Barbosa, A.C., and Olson, E.N. 2006. Coactivation of MEF2 by the SAP domain proteins myocardin and MASTR. Mol. Cell. 23:83-96.

21. Du, K.L., et al. 2003. Myocardin is a critical serum response factor cofactor in the transcriptional program regulating smooth muscle cell differentiation. Mol. Cell. Biol. 23:2425-2437.

22. Wang, D., et al. 2001. Activation of cardiac gene expression by myocardin, a transcriptional cofactor for serum response factor. Cell. 105:851-862.

23. Yoshida, T., et al. 2003. Myocardin is a key regulator of CArG-dependent transcription of multiple 
smooth muscle marker genes. Circ. Res. 92:856-864.

24. Chen, J., Kitchen, C.M., Streb, J.W., and Miano, J.M. 2002. Myocardin: a component of a molecular switch for smooth muscle differentiation. J. Mol. Cell. Cardiol. 34:1345-1356.

25. Li, S., Wang, D.Z., Wang, Z., Richardson, J.A., and Olson, E.N. 2003. The serum response factor coactivator myocardin is required for vascular smooth muscle development. Proc. Natl. Acad. Sci. U. S. A. 100:9366-9370.

26. Wang, D.Z., et al. 2002. Potentiation of serum response factor activity by a family of myocardin-related transcription factors. Proc. Natl. Acad. Sci. U. S. A. 99:14855-14860.

27. Miralles, F., Posern, G., Zaromytidou, A.I., and Treisman, R. 2003. Actin dynamics control SRF activity by regulation of its coactivator MAL. Cell. 113:329-342.

28. Du, K.L., et al. 2004. Megakaryoblastic leukemia factor-1 transduces cytoskeletal signals and induces smooth muscle cell differentiation from undifferentiated embryonic stem cells. J. Biol. Chem. 279:17578-17586.

29. Li, J., et al. 2005. Myocardin-related transcription factor $\mathrm{B}$ is required in cardiac neural crest for smooth muscle differentiation and cardiovascular development. Proc. Natl. Acad. Sci. U. S. A. 102:8916-8921.

30. Oh, J., Richardson, J.A., and Olson, E.N. 2005. Requirement of myocardin-related transcription factor-B for remodeling of branchial arch arteries and smooth muscle differentiation. Proc. Natl. Acad. Sci.U.S. A. 102:15122-15127.

31. Wei, K., Che, N., and Chen, F. 2007. Myocardinrelated transcription factor $\mathrm{B}$ is required for normal mouse vascular development and smooth muscle gene expression. Dev. Dyn. 236:416-425.
32. Sun, Y., et al. 2006. Acute myeloid leukemia-associated Mkl1 (Mrtf-a) is a key regulator of mammary gland function. Mol. Cell. Biol. 26:5809-5826.

33. Schwenk, F., Baron, U., and Rajewsky, K. 1995. A cretransgenic mouse strain for the ubiquitous deletion of loxP-flanked gene segments including deletion in germ cells. Nucleic Acids Res. 23:5080-5081.

34. Chai, Y., et al. 2000. Fate of the mammalian cranial neural crest during tooth and mandibular morphogenesis. Development. 127:1671-1679.

35. Soriano, P. 1999. Generalized lacZ expression with the ROSA26 Cre reporter strain. Nat. Genet. 21:70-71.

36. Li, J., Chen, F., and Epstein, J.A. 2000. Neural crest expression of Cre recombinase directed by the proximal Pax3 promoter in transgenic mice. Genesis. 26:162-164.

37. Gittenberger-de Groot, A.C., van Ertbruggen, I., Moulaert, A.J., and Harinck, E. 1980. The ductus arteriosus in the preterm infant: histologic and clinical observations. J. Pediatr. 96:88-93.

38. Schneider, D.J., and Moore, J.W. 2006. Patent ductus arteriosus. Circulation. 114:1873-1882.

39. Waldo, K.L., et al. 2005. Secondary heart field contributes myocardium and smooth muscle to the arterial pole of the developing heart. Dev. Biol. 281:78-90.

40. Owens, G.K., Kumar, M.S., and Wamhoff, B.R. 2004. Molecular regulation of vascular smooth muscle cell differentiation in development and disease. Physiol. Rev. 84:767-801.

41. Wang, Z., et al. 2004. Myocardin and ternary complex factors compete for SRF to control smooth muscle gene expression. Nature. 428:185-189.

42. Loeys, B.L., et al. 2005. A syndrome of altered cardiovascular, craniofacial, neurocognitive and skeletal development caused by mutations in TGFBR1 or TGFBR2. Nat. Genet. 37:275-281.

43. Khau Van Kien, P., et al. 2005. Mapping of familial thoracic aortic aneurysm/dissection with patent ductus arteriosus to $16 \mathrm{p} 12.2$-p13.13. Circulation. 112:200-206

44. Testa, G., et al. 2003. Engineering the mouse genome with bacterial artificial chromosomes to create multipurpose alleles. Nat. Biotechnol. 21:443-447.

45. Lepore, J.J., et al. 2006. GATA-6 regulates semaphorin $3 \mathrm{C}$ and is required in cardiac neural crest for cardiovascular morphogenesis. J. Clin. Invest. 116:929-939.

46. Morrisey, E.E., et al. 1998. GATA6 regulates HNF4 and is required for differentiation of visceral endoderm in the mouse embryo. Genes Dev. 12:3579-3590.

47. Lakso, M., et al. 1992. Targeted oncogene activation by site-specific recombination in transgenic mice. Proc. Natl. Acad. Sci. U. S. A. 89:6232-6236.

48. Jiang, X., Rowitch, D.H., Soriano, P., McMahon, A.P., and Sucov, H.M. 2000. Fate of the mammalian cardiac neural crest. Development. 127:1607-1616.

49. Gavrieli, Y., Sherman, Y., and Ben-Sasson, S.A. 1992. Identification of programmed cell death in situ via specific labeling of nuclear DNA fragmentation. J. Cell Biol. 119:493-501.

50. Lepore, J.J., Cappola, T.P., Mericko, P.A., Morrisey, E.E., and Parmacek, M.S. 2005. GATA-6 regulates genes promoting synthetic functions in vascular smooth muscle cells. Arterioscler. Thromb. Vasc. Biol. 25:309-314.

51. Huang, J., Zhao, Q., Mooney, S.M., and Lee, F.S. 2002. Sequence determinants in hypoxia-inducible factor-1alpha for hydroxylation by the prolyl hydroxylases PHD1, PHD2, and PHD3. J. Biol. Chem. 277:39792-39800. 